\title{
Clinical study on the association between pregnancy-induced hypertension and insulin resistance
}

\author{
ZHIFANG CHEN, WEILING LIU, XIAOQIN SUN and LINGLING ZHU \\ Department of Obstetrics and Gynecology, Nantong Maternity and Child Health Care Hospital Affiliated \\ to Nantong University, Nantong, Jiangsu 226018, P.R. China
}

Received September 15, 2015; Accepted October 28, 2016

DOI: 10.3892/etm.2017.4169

\begin{abstract}
The aim of the present study was to explore the association between pregnancy-induced hypertension (PIH) and insulin resistance (IR). A total of 50 cases of PIH and 50 healthy pregnant women with a similar gestational age were enrolled. The hyperinsulinemic-euglycemic clamp technique was used to evaluate the degree of IR and all 100 subjects were divided into an IR and a non-IR group accordingly. Subsequently, the correlation between the systolic or diastolic blood pressure was assessed; furthermore, a homeostasis model assessment of IR (HOMA-IR), a HOMA of the insulin sensitivity index (HOMA-ISI) and a HOMA of $\beta$ cell function (HOMA- $\beta \%$ ) were performed. Moreover, the effect of IR on $\mathrm{PIH}$ was assessed and the protein expression of insulin receptor substrate (IRS)-1, phosphorylated (p)-IRS-1, AKT and p-AKT were detected in the placental plasma by western blot analysis. The results showed that in the PIH group, the p-IRS-1/IRS-1 and $\mathrm{p}-\mathrm{AKT} / \mathrm{AKT}$ ratios were decreased compared with those in the control group. Blood flow parameters, including perfusion index, retinal resistive index and systolic maximum velocity/end-diastolic velocity ratio in the IR group were higher, while time averaged velocity was lower compared with that in the non-IR group. Furthermore, the HOMA-ISI and HOMA- $\beta \%$ were decreased, while the HOMA-IR was increased in the PIH group compared to that in the control group; alongside the blockage of the insulin signaling pathway, these factors may therefore cause PIH. The present study may provide novel therapeutic approaches for PIH.
\end{abstract}

\section{Introduction}

The clinical term for gestational hypertension is clinically pregnancy-induced hypertension (PIH) syndrome (1). It is a

Correspondence to: Miss Zhifang Chen, Department of Obstetrics and Gynecology, Nantong Maternity and Child Health Care Hospital Affiliated to Nantong University, 399 Century Avenue, Nantong, Jiangsu 226018, P.R. China

E-mail: chenzhifangczf@163.com

Key words: HOMA-IR, HOMA-ISI, HOMA- $\beta \%$, insulin resistance, pregnancy-induced hypertension distinctive and common disease during pregnancy which is characterized by high blood pressure, edema, proteinuria, convulsions, coma, heart failure and renal failure (2). This disease severely affects maternal and child health and is one of the leading causes of maternal and neonatal mortality (3). Insulin resistance (IR) refers to a physiological condition in which cells fail to take in glucose, amino acids and fatty acids and cause high blood glucose (4). To maintain stable blood sugar levels, $\beta$ cells in the pancreas subsequently increase their production of insulin, which results in hyperinsulinemia (5). A study has shown that in a non-diabetic population, systolic and diastolic blood pressure were higher in the high insulin group when compared with that in a normal insulin group (6). IR is positively correlated with the prevalence of hypertension.

Insulin receptor substrate (IRS) can be affected by insulin receptor tyrosine kinase (7). IRS-1-4 are known insulin receptor substrates. Phosphorylation of IRS-1 can activate downstream effectors to then regulate the insulin signal transduction pathways (8). IRS-1 is phosphorylated by tyrosine kinase and then activates the downstream AKT (9). Therefore, IRS-1 and AKT are two important kinases in the insulin signal transduction pathways.

The aim of the present study was to explore the association between PIH and IR, which may provide a reasonable solution for the clinical treatment of PIH.

\section{Materials and methods}

Ethics statement. The present study was approved by the Ethics Committee of Nantong Maternity and Child Health Care Hospital (Nantong, China; no. 20130215). All subjects were provided with the relevant information (purpose and content) of the present study and informed consent was obtained from all participants.

Antibodies and reagents. Human triglyceride (TG) ELISA kit, human total cholesterol (TC) ELISA kit, human low density lipoprotein (LDL) ELISA kit, human insulin ELISA kit, human high density lipoprotein (HDL) kit and fasting blood glucose (FBG) kit were purchased from Sigma-Aldrich (Merck Millipore, Darmstadt, Germany). human urine protein C ELISA kit, human blood urea nitrogen (BUN) ELISA kit and human serum creatinine (Scr) ELISA kit were purchased from R\&D Systems Inc., (Minneapolis, MN, USA). Human apolipoprotein 
A-1 (apoA-1) ELISA and apolipoprotein B (apoB) ELISA kits were purchased from Abcam (Cambridge, MA, USA). Bicinchoninic acid (BCA) kit, enhanced chemiluminescence (ECL) chemiluminescence kit and $\beta$-actin antibody (AF0003) were purchased from Beyotime Institute of Biotechnology (Jiangsu, China). AKT antibody (sc-5298), phospho-AKT antibody (sc-293125), IRS-1 antibody (sc-515017) and phospho-IRS-1 antibody (sc-33956) were purchased from Santa Cruz Biotechnology Inc., (Dallas, TX, USA).

Subjects. A total of 50 pregnant women with hypertension (average gestational age, 230.12 \pm 18.9 days) were enrolled at Nantong Maternity and Child Health Care Hospital (Nantong, China) between May and October of 2013 as the pregnancy-induced hypertension group (PIH group). Hypertension was diagnosed according to the guidelines of the National Hypertension Education Project Group (National High Blood Pressure Working Group) from 2000 and Williams Obstetrics 21st edition $(10,11)$. Another 50 healthy pregnant women who underwent regular obstetric examination at Nantong Maternity and Child Health Care Hospital at the same time (average gestational age, $228.5 \pm 15.5$ days) were enrolled as the normal group (control group).

After $12 \mathrm{~h}$ of fasting overnight, $5 \mathrm{ml}$ venous blood was collected. One aliquot of the blood was placed in a centrifuge tube containing heparin, mixed and centrifuged at 5,000 $\mathrm{x} g$ for $10 \mathrm{~min}$. The supernatant, i.e., plasma, was obtained for western blot analysis. Another aliquot of the venous blood was placed in a centrifuge tube without heparin. After blood coagulation, the supernatant obtained after removal of condensed blood clots was serum, which was used for determining TG, TC, LDL, HDL, FBG and fasting insulin (FINS). Early morning urine was obtained for detecting urine protein, blood urea nitrogen (BUN) and serum creatinine (Scr).

All subjects had a single fetus and did not suffer from gestational diabetes, but experienced obstetric complications, including impaired sugar tolerance, premature rupture of membranes and placenta previa as well as a history of medical complications, including chronic hypertension, diabetes mellitus, heart disease and nephropathy.

Determination of $T G, T C, L D L, H D L$, apoA-1, apoB, urine protein, BUN and Scr. The concentrations of TG, TC, LDL, HDL, apoA-1, apoB, urine protein, BUN and Scr were measured in the serum using the respective commercial kits. All measurements were performed according to the manufacturer's instructions. An automatic biochemistry analyzer (model 7600; Hitachi, Tokyo, Japan) was used to record various indexes. TG, TC and LDL levels were measured via the enzymatic method. HDL levels were detected by the polyanion polymer/detergent assay. Levels of apoA-1 apoB were determined via the immunoturbidimetric method. Urine protein, BUN and Scr contents were detected by the enzymatic method. Creatinine clearance rate (Ccr) was calculated as $\mathrm{Ccr}=[40$-age (years) $] \mathrm{x}$ weight $(\mathrm{kg}) /[0.85 \mathrm{x} \mathrm{Scr}$ $(\mu \mathrm{mol} / \mathrm{l})]$.

Measurement of FBG and FINS. FBG was detected by the glucose oxidase method following the manufacturer's instructions. The glucose concentration was determined by the absorbance of known concentrations of control blue substances at $625 \mathrm{~nm}$ wavelength.

The blood insulin level was detected by an enzyme-linked immune chemiluminescence method with a human insulin ELISA kit according to the manufacturer's instructions. To detect FINS, samples, standard preparation and horseradish peroxidase (HRP)-labeled detection antibody were added in micropores coated with capture antibody one by one. After incubation at $37^{\circ} \mathrm{C}$ for $30 \mathrm{~min}$, pores were washed thoroughly and antibodies were visualized with a 3,3',5,5'-tetramethylbenzidine substrate. Optical density values were measured at $450 \mathrm{~nm}$ with automatic chemiluminescence analyzer (DTX880 Multimode Plate Reader; Beckman Coulter, Inc., Brea, CA, USA), from which the insulin content in samples was calculated.

Homeostasis model assessment (HOMA). Islet $\beta$ cell function and the degree of insulin resistance were evaluated via HOMA using the following formulas: HOMA-IR=FINS x FBG/22.5; HOMA-insulin sensitivity index (ISI) $=$ In $[22.5 /(\mathrm{FBG} \times \mathrm{FINS})]$; and HOMA- $\beta$ cell function $(\beta \%)=20 \mathrm{x}$ FINS/(FBG-3.5).

Determination of placental bed blood flow parameters. Placental bed blood flow parameters, including the ratio of the systolic maximum velocity and the end-diastolic velocity (S/D), pulsatility index (PI), resistance index (RI) and time averaged velocity (TAV) were measured with YF-E820 color Doppler ultrasonic diagnosis apparatus (Sichuan Yufeng Technology Development Co., Ltd., Mianyang, China) at the 32nd week of gestation.

Western blot analysis. After fasting for $12 \mathrm{~h}$ overnight, $4 \mathrm{ml}$ venous blood was collected. The serum was separated and stored at $-80^{\circ} \mathrm{C}$ until analysis. The protein levels of IRS-1, p-IRS-1, AKT and p-AKT were measured by western blot analysis as described previously (9). In brief, serum protein content was quantified by a BCA kit. A total of $60 \mu \mathrm{g}$ serum protein was separated by $8-12 \%$ SDS-PAGE and transferred onto a polyvinylidene fluoride membrane (EMD Millipore, Billerica, MA, USA). Following blocking in 5\% non-fat milk diluted with Tris-buffered saline with Tween-20 (TBST; Tris-HCl 20 mM, NaCl 150 mM, pH 7.4, 0.1\% Tween-20) at room temperature for $45 \mathrm{~min}$ and then probed overnight at $4^{\circ} \mathrm{C}$ with the following primary antibodies: $\beta$-actin, IRS-1, AKT (1:4,000 dilution), p-IRS-1 and p-AKT (1:1,000 dilution). Following washing with TBST, membranes were incubated with the appropriate secondary antibodies for $1 \mathrm{~h}$ at room temperature. The bands were developed using an ECL chemiluminescence kit and then visualized by exposure to X-ray film (Kodak, Rochester, NY, USA). The results were scanned and analyzed using ImageJ software (version 1.48; National Institutes of Health, Bethesda, MD, USA).

Evaluation of the degree of IR. The euglycemic-hyperinsulinemic clamp method was used to evaluate the degree of IR. Intravenous injection of insulin can cause hyperproinsulinemia. The insulin infusion speed was $127.6 \mathrm{mU} / \mathrm{m}^{3} / \mathrm{min}$ at the beginning, was reduced to $40 \mathrm{mU} / \mathrm{m}^{3} / \mathrm{min} 10 \mathrm{~min}$ later and maintained at this speed until end of the test. The experiment 
lasted $2 \mathrm{~h}$. When insulin was infused for $20 \mathrm{~min}$, the blood insulin concentrations increased from $14 \pm 1$ to $105 \pm 5 \mathrm{mU} / \mathrm{l}$ and reached a steady state. The glucose was infused 4 min after insulin infusion and the glucose levels were monitored every $5 \mathrm{~min}$. The infusion speed and concentration of glucose were adjusted to prevent low blood glucose. The production of liver endogenous glucose was inhibited by $90 \%$ when the concentration of blood insulin was $>50 \mathrm{mU} / 1$. The blood glucose was maintained at a normal level via mediating the exogenous glucose at this time. The infusion amount of exogenous glucose was equal to the processed amount of glucose by the body through the action of insulin. So the body's sensitivity to insulin was determined by the ratio of exogenous glucose and blood insulin. Patients were diagnosed as having IR when the utilization of glucose was $<7.24 \mathrm{mg} / \mathrm{kg} / \mathrm{min}$.

Statistical analysis. Values are expressed as the mean \pm standard deviation. The correlation of p-IRS-1 and p-AKT with HOMA-IR, HOMA-ISI and HOMA- $\beta \%$ was analyzed using the Spearman Rank Correlation test. Comparisons between 2 groups were analyzed using Student's $t$-test and the differences among 3 groups were analyzed by analysis of variance followed by a post hoc comparison using the least significant difference test (SPSS 19.0; SPSS IBM, Armonk, NY, USA). $\mathrm{P}<0.05$ was considered to indicate a statistically significant difference.

\section{Results}

Clinical data of healthy control and PIH groups. As shown in Table I, there was no significant differences in average age between the two groups $(\mathrm{P}>0.05)$; however, body mass index (BMI), SBP level and DBP level in the PIH group were significantly higher than those in the control group $(\mathrm{P}<0.01)$.

Differences in FBG, FINS, HOMA-IR, HOMA-ISI and HOMA- $\beta \%$ between healthy control and PIH groups. As shown in Table II, compared with the healthy control group, the FBG, FINS and HOMA-IR in the PIH group were significantly increased, while the HOMA-ISI and HOMA- $\beta \%$ were significantly reduced compared with those in the control group $(\mathrm{P}<0.05)$.

Correlation of p-IRS-1 and p-AKT with HOMA-IR, HOMA-ISI and HOMA- $\beta \%$ in the PIH group. As shown in Table III, the correlation analyses revealed a negative correlation between p-IRS-1 and HOMA-IR $(\mathrm{P}<0.01)$ but a positive correlation between p-IRS-1 and HOMA-ISI $(\mathrm{P}<0.05)$. Similarly, there was a negative correlation between $\mathrm{p}-\mathrm{AKT}$ and HOMA-IR $(\mathrm{P}<0.01)$ but a positive correlation between $\mathrm{p}-\mathrm{AKT}$ and HOMA-ISI $(\mathrm{P}<0.05)$.

Differences in placental bed blood flow parameters between $I R$ and non-IR groups. Dlood flow parameters, including PI, RI, S/D and TAV, were determined in all subjects using a color Doppler ultrasonic diagnosis apparatus. As shown in Table IV, the results revealed that the PI, RI and S/D in the IR group were significantly higher than those in the non-IR group $(\mathrm{P}<0.05)$, while the TAV in the IR group was significantly lower than that in the non-IR group $(\mathrm{P}<0.05)$.
Differences in protein expression of IRS-1, p-IRS-1, AKT and $p-A K T$ in the plasma between healthy control and PIH groups. As shown in Fig. 1, the levels of total IRS-1 and AKT were not significantly different between healthy control and PIH groups $(\mathrm{P}>0.05)$, but the levels of $\mathrm{p}-\mathrm{IRS}-1$ and $\mathrm{p}-\mathrm{AKT}$ and the $\mathrm{p}$-IRS-1/IRS-1 and p-AKT/AKT ratios in the PIH group were significantly reduced compared with those in the healthy control group $(\mathrm{P}<0.05)$.

\section{Discussion}

For fetal development and post-natal breastfeeding, the secretion of cytokines (such as leptin, adiponectin, visceral fatty acids and resistin) by adipose tissue increases under the influence of progesterone and placental lactogen, but the ability to synthesize them decreases, so that normal pregnant women present with hyperlipidemia (12-15). In the present study, serum TC, TG and LDL increased in normal pregnant women, but HDL and apoA-1 decreased, so that each index was maintained at a normal level (16-18). However, the levels of TG, LDL and apoB were higher in pregnant women with gestational hypertension compared with the normal group, but HDL and apoA-1 levels were diminished in pregnant women with gestational hypertension. The results also demonstrated that urine protein, BUN and Scr levels were higher in patients with gestational hypertension compared with the normal group. All these findings suggested that gestational hypertension led to abnormal lipid metabolism $(19,20)$.

Studies have confirmed that insulin regulates lipid metabolism and carbohydrate uptake $(21,22)$. Insulin resistance reduces the sensitivity of adipocytes, myocytes and hepatocytes to insulin, promotes glucose uptake, weakens the antilipolytic capability, further resulting in compensatory hyperinsulinemia and the release of fatty acids into the blood to then cause hyperglycemia combined with hyperinsulinism and lipid metabolism disorder (23). The body is insulin resistant through signal transduction by the insulin receptor and autophosphorylation. At least eight tyrosine residues on IRS-1 can be phosphorylated by insulin receptor tyrosine kinase. p-IRS-1 can bind to and activate downstream effectors, participates in the signal transduction of various hormones and cytokines, has an important role in cell growth, differentiation and metabolism, and simultaneously maintains a metastable state of insulin and blood sugar levels (24-26). The increase in p-IRS-1 contributes to the transduction of insulin signaling, weakens insulin resistance and reduces blood sugar. The phosphoinositide-3 kinase (PI3K)-AKT signaling pathway has a crucial effect on mediating insulin-stimulated glucose transport. The decreased p-AKT level can enhance glucose transport capacity, weaken insulin resistance and diminish free blood sugar levels. In addition, PI3K-AKT is involved in free fatty acid synthesis (27). Simultaneously, free fatty acid suppresses insulin binding to its receptor and aggravates insulin resistance (28). The results of the present study showed that the BMI, TG, TC, FBG and FINS were greater in the PIH group than those in the normal control group. Compared with the normal control group, HOMA-IR was increased and HOMA-ISI was decreased in the PIH group. Phosphorylation levels of IRS-1 and AKT were significantly lower in the PIH group compared with those in the normal 
Table I. Clinical data on the healthy control and PIH groups.

\begin{tabular}{|c|c|c|c|c|c|c|}
\hline \multirow[b]{2}{*}{ Group } & \multirow[b]{2}{*}{$\mathrm{N}$} & \multirow[b]{2}{*}{ Age (years) } & \multicolumn{2}{|c|}{ BMI $\left(\mathrm{kg} / \mathrm{m}^{2}\right)$} & \multirow[b]{2}{*}{$\mathrm{SBP}(\mathrm{mmHg})$} & \multirow[b]{2}{*}{$\mathrm{DBP}(\mathrm{mmHg})$} \\
\hline & & & 4 weeks & 32 weeks & & \\
\hline Control group & 50 & $26.8 \pm 3.2$ & $20.74 \pm 2.32$ & $26.6 \pm 3.34$ & $112.6 \pm 12.5$ & $75.6 \pm 7.4$ \\
\hline PIH group & 50 & $27.2 \pm 2.5$ & $26.74 \pm 1.63$ & $32.5 \pm 4.62$ & $162.7 \pm 10.5$ & $108.5 \pm 6.9$ \\
\hline P-value & & 0.854 & 0.023 & 0.038 & 0.048 & 0.006 \\
\hline
\end{tabular}

Values are expressed as the mean \pm standard deviation. BMI, body-mass index; SBP, systolic blood pressure; BDP, diastolic blood pressure.

Table II. Differences in parameters between healthy control and PIH groups. Significant differences in FBG, FINS, HOMA-IR, HOMA-ISI and HOMA- $\beta \%$ were observed.

\begin{tabular}{lccr}
\hline Parameter & Control group $(\mathrm{n}=50)$ & PIH group $(\mathrm{n}=50)$ & P-value \\
\hline FBG (pmol/l) & $45.3 \pm 8.6$ & $85.9 \pm 8.2$ & $<0.001$ \\
FINS (mmol/l) & $8.23 \pm 1.92$ & $15.78 \pm 1.35$ & 0.022 \\
HOMA-IR & $1.63 \pm 0.58$ & $4.34 \pm 0.76$ & 0.014 \\
HOMA- $\beta \%$ & $225.49 \pm 19.02$ & $136.58 \pm 25.42$ & $<0.001$ \\
HOMA- ISI & $-0.25 \pm 0.02$ & $-1.49 \pm 0.13$ & $<0.001$ \\
TG (mmol/l) & $2.12 \pm 0.72$ & $3.35 \pm 1.43$ & 0.035 \\
TC (mmol/l) & $5.89 \pm 1.35$ & $5.96 \pm 1.02$ & 0.896 \\
LDL (mmol/l) & $2.09 \pm 0.52$ & $3.02 \pm 0.42$ & 0.014 \\
HDL (mmol/l) & $2.55 \pm 0.32$ & $1.94 \pm 0.25$ & 0.028 \\
apoB (pmol/l) & $1.01 \pm 0.04$ & $1.18 \pm 0.06$ & 0.041 \\
apoA-1 (pmol/l) & $2.69 \pm 0.12$ & $2.01 \pm 0.11$ & 0.036 \\
Urine protein $(\mathrm{g} / 24 \mathrm{~h})$ & $0.12 \pm 0.24$ & $1.36 \pm 0.42$ & 0.002 \\
BUN (mmol/l) & $5.10 \pm 1.03$ & $7.73 \pm 1.15$ & 0.039 \\
Scr $(\mu \mathrm{mol} / \mathrm{l})$ & $102.32 \pm 10.51$ & $159.15 \pm 17.32$ & 0.008 \\
Ccr (ml/min) & $90.23 \pm 12.32$ & $55.33 \pm 8.36$ & 0.004 \\
\hline
\end{tabular}

Values are expressed as the mean \pm standard deviation. TG, triglyceride; TC, total cholesterol; LDL, low-density lipoprotein; HDL, high-density lipoprotein; FBG, fasting blood glucose; FINS, fasting insulin; BUN, blood urea nitrogen; Scr, serum creatinine; apo, apolipoprotein; HOMA-IR homeostasis model assessment of IR; HOMA-ISI, HOMA of insulin sensitivity index; HOMA- $\beta \%$, HOMA of $\beta$-cell function; Scr, serum creatinine; Ccr, creatinine clearance rate.

Table III. Analysis of the correlation of p-IRS-1 and p-AKT with HOMA-IR and HOMA-ISI in PIH group.

\begin{tabular}{|c|c|c|c|c|}
\hline \multirow[b]{2}{*}{ Parameter } & \multicolumn{2}{|c|}{ p-IRS-1 } & \multicolumn{2}{|c|}{$\mathrm{p}-\mathrm{AKT}$} \\
\hline & Correlation coefficient & P-value & Correlation coefficient & P-value \\
\hline HOMA-IR & -0.473 & $<0.01$ & -0.520 & $<0.01$ \\
\hline HOMA-ISI & 0.342 & $<0.05$ & 0.487 & $<0.05$ \\
\hline
\end{tabular}

Spearman's rank correlation coefficients are shown. HOMA-IR homeostasis model assessment of IR; HOMA-ISI, HOMA of insulin sensitivity index; p-IRS-1, phosphorylated insulin resistance substrate.

control group. Moreover, p-IRS-1 and p-AKT were negatively correlated with HOMA-IR, while being positively associated with HOMA-ISI. The above results indicated that PIH possibly occurs due to the following reasons: Downregulation of IRS-1 phosphorylation leads to reduced activation of signaling pathways downstream of insulin, which causes blood sugar levels to increase. The reduction in AKT phosphorylation levels induces a decline in glucose transporter function, which 
Table IV. Differences in placental bed blood flow parameters between IR and non-IR groups.

\begin{tabular}{|c|c|c|c|c|c|}
\hline Group & $\mathrm{N}$ & RI & PI & $\mathrm{S} / \mathrm{D}$ & TAV \\
\hline Non-IR group & 30 & $0.43 \pm 0.05$ & $0.62 \pm 0.09$ & $1.75 \pm 0.25$ & $15.33 \pm 1.72$ \\
\hline IR group & 70 & $0.67 \pm 0.12$ & $0.92 \pm 0.07$ & $2.78 \pm 0.36$ & $9.86 \pm 2.47$ \\
\hline P-value & & $<0.05$ & $<0.05$ & $<0.05$ & $<0.05$ \\
\hline
\end{tabular}

Values are expressed as the mean \pm standard deviation. IR, insulin resistance; S/D, end-diastolic velocity; PI, pulsatility index; RI, resistance index; TAV, time averaged velocity.

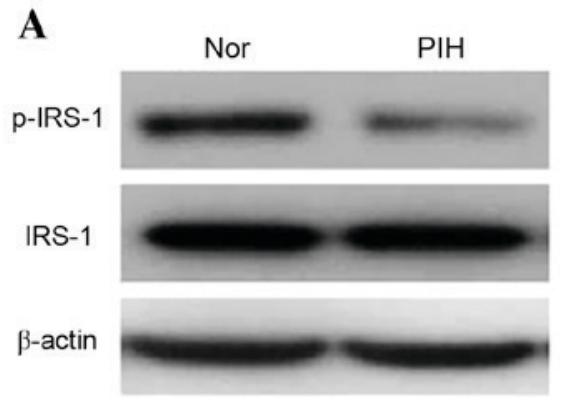

C

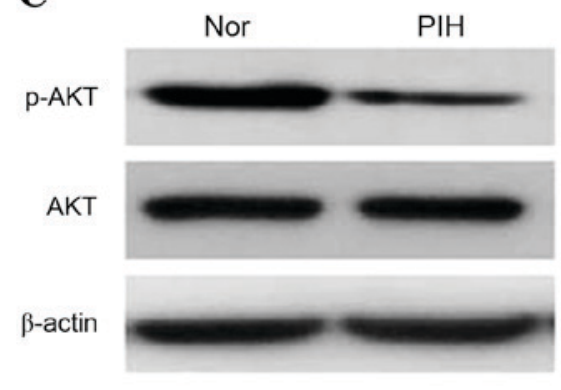

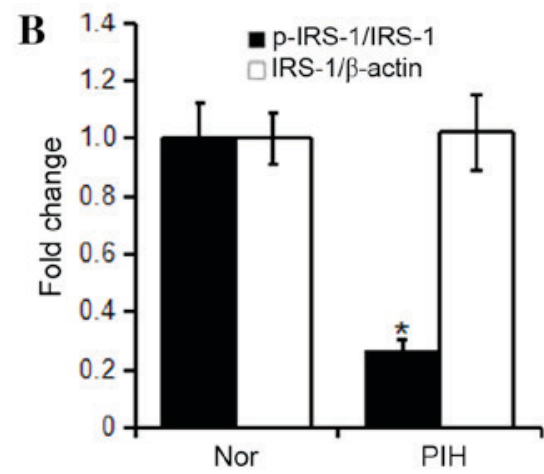

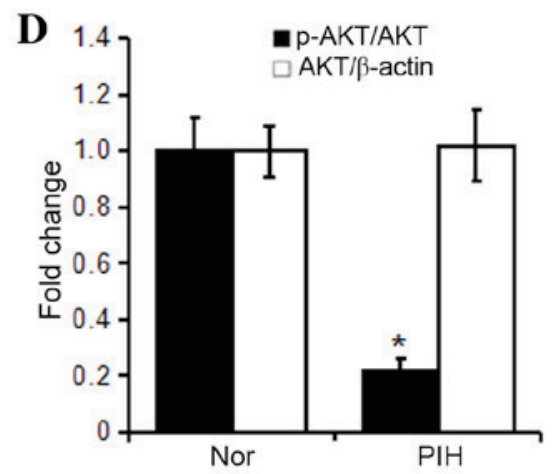

Figure 1. Expression of (A and B) IRS-1 and p-IRS-1, and (C and D) AKT and p-AKT in the PIH and healthy control groups. Values are expressed as the mean \pm standard deviation $(\mathrm{n}=50)$. ${ }^{*} \mathrm{P}<0.05$ vs. Nor. $\mathrm{p}$-IRS, phosphorylated insulin resistance substrate; PIH, pregnancy-induced hypertension; Nor, healthy control group.

results in increased blood sugar levels and lipid metabolism disorder. Moreover, the decreased phosphorylation levels of IRS-1 and AKT elevate HOMA-IR and decrease HOMA-ISI, further suggesting that insulin resistance is one of the major reasons for PIH occurrence.

In conclusion, the present study indicated that insulin resistance may be a key factor causing PIH and may provide novel therapeutic approaches for PIH.

\section{References}

1. Salge AK, Xavier RM, Ramalho WS, Rocha EL, Coelho AS, Guimarães JV, Siqueira KM, Abdalla DR, Michelin MA and Murta EF: Placental and umbilical cord macroscopic changes associated with fetal and maternal events in the hypertensive disorders of pregnancy. Clin Exp Obstet Gynecol 40: 198-202, 2013.

2. Flores KF, Robledo CA, Hwang BS, Leishear K, Laughon Grantz K and Mendola P: Does maternal asthma contribute to racial/ethnic disparities in obstetrical and neonatal complications? Ann Epidemiol 25: 392-397, 2015.
3. Yang JJ, Lee SA, Choi JY, Song M, Han S, Yoon HS, Lee Y, Oh J, Lee JK and Kang D: Subsequent risk of metabolic syndrome in women with a history of preeclampsia: Data from the health examinees study. J Epidemiol 25: 281-288, 2015.

4. Walker CK, Krakowiak P, Baker A, Hansen RL, Ozonoff S and Hertz-Picciotto I: Preeclampsia, placental insufficiency, and autism spectrum disorder or developmental delay. JAMA Pediatr 169: 154-162, 2015.

5. Katulski K, Czyzyk A, Podfigurna-Stopa A, Genazzani AR and Meczekalski B: Pregnancy complications in polycystic ovary syndrome patients. Gynecol Endocrinol 31: 87-91, 2015.

6. Rahmanian M, Salari Z, Mirmohammadkhani M and Ghorbani R: Is the sex hormone binding globulin related to preeclampsia independent of insulin resistance? J Pak Med Assoc 64: 640-643, 2014.

7. Tuuri AL, Jauhiainen MS, Tikkanen MJ and Kaaja RJ: Systolic blood pressure and fatty acid-binding protein 4 predict pregnancy-induced hypertension in overweight nulliparous women. Placenta 35: 797-801, 2014.

8. Abhari FR, Ghanbari Andarieh M, Farokhfar A and Ahmady S: Estimating rate of insulin resistance in patients with preeclampsia using HOMA-IR index and comparison with nonpreeclampsia pregnant women. Biomed Res Int 2014: 140851, 2014.

9. Zhang H, Zhao D, Shen J, Zhou X, Chen W and Jiang S: Evaluation of oral glucose tolerance test, $\beta$-cell function and adverse obstetric outcomes. Biomed Rep 1: 807-811, 2013. 
10. Report of the national high blood pressure education program working group on high blood pressure in pregnancy. Am J Obstet Gynecol 183: S1-S22, 2000.

11. Cunningham FG, Gant NF, Leveno KJ, Gilstrap LC, Hauth JC, Wenstrom KD, Werner CL and Cox SM: Williams Obstetrics, 21st edition. McGraw-Hill, New York, NY, 2001.

12. Sahasrabuddhe A, Pitale S, Raje D and Sagdeo MM: Cord blood levels of insulin and glucose in full-term pregnancies. J Assoc Physicians India 61: 378-382, 2013.

13. Mastrogiannis DS, Spiliopoulos M, Mulla W and Homko CJ: Insulin resistance: The possible link between gestational diabetes mellitus and hypertensive disorders of pregnancy. Curr Diab Rep 9: 296-302, 2009.

14. Garcés MF, Peralta JJ, Ruiz-Linares CE, Lozano AR, Poveda NE, Torres-Sierra AL, Eslava-Schmalbach JH, Alzate JP, Sánchez AY, Sanchez E, et al: Irisin levels during pregnancy and changes associated with the development of preeclampsia. J Clin Endocrinol Metab 99: 2113-2119, 2014.

15. Huda SS, Forrest R, Paterson N, Jordan F, Sattar N and Freeman DJ: In preeclampsia, maternal third trimester subcutaneous adipocyte lipolysis is more resistant to suppression by insulin than in healthy pregnancy. Hypertension 63: 1094-1101, 2014.

16. Tran M, Gallo LA, Hanvey AN, Jefferies AJ, Westcott KT, Cullen-McEwen LA, Gardner DK, Moritz KM and Wlodek ME: Embryo transfer cannot delineate between the maternal pregnancy environment and germ line effects in the transgenerational transmission of disease in rats. Am J Physiol Regul Integr Comp Physiol 306: R607-R618, 2014.

17. Valdés E, Sepúlveda-Martínez A, Manukián B and Parra-Cordero M: Assessment of pregestational insulin resistance as a risk factor of preeclampsia. Gynecol Obstet Invest 77: 111-116, 2014.

18. Lampinen KH, Rönnback M, Groop PH, Nicholls MG, Yandle TG and Kaaja RJ: Increased plasma norepinephrine levels in previously pre-eclamptic women. J Hum Hypertens 28: 269-273, 2014

19. Scioscia M, Nigro $M$ and Montagnani M: The putative metabolic role of d-chiro inositol phosphoglycan in human pregnancy and preeclampsia. J Reprod Immunol 101-102: 140-147, 2014.
20. Tuuri AL, Jauhiainen MS, Ehnholm CP, Tikkanen MJ, Nicholls MG and Kaaja RJ: Elevated serum angiopoietin-like protein 6 in women with subsequent pregnancy-induced hypertension: A preliminary study. Hypertens Pregnancy 32: 203-213, 2013.

21. Harper LM, Renth A, Cade WT, Colvin R, Macones GA and Cahill AG: Impact of obesity on maternal and neonatal outcomes in insulin-resistant pregnancy. Am J Perinatol 31: 383-388, 2014.

22. Romero J and Spinedi E: Two-hour insulinemia after oral glucose overload and women at risk of pregnancy-induced hypertensive disorders. Hypertens Pregnancy 32: 355-366, 2013.

23. Oxenkrug G: Insulin resistance and dysregulation of tryptophan-kynurenine and kynurenine-nicotinamide adenine dinucleotide metabolic pathways. Mol Neurobiol 48: 294-301, 2013.

24. Duehlmeier R, Noldt S and Ganter M: Pancreatic insulin release and peripheral insulin sensitivity in German black headed mutton and Finish Landrace ewes: Evaluation of the role of insulin resistance in the susceptibility to ovine pregnancy toxemia. Domest Anim Endocrinol 44: 213-221, 2013.

25. Duehlmeier R, Fluegge I, Schwert B and Ganter M: Insulin sensitivity during late gestation in ewes affected by pregnancy toxemia and in ewes with high and low susceptibility to this disorder. J Vet Intern Med 27: 359-366, 2013.

26. Kayemba-Kay's S, Peters C, Geary MP, Hill NR, Mathews DR and Hindmarsh PC: Maternal hyperinsulinism and glycaemic status in the first trimester of pregnancy are associated with the development of pregnancy-induced hypertension and gestational diabetes. Eur J Endocrinol 168: 413-418, 2013.

27. Smith GN, Pudwell J, Walker M and Wen SW: Risk estimation of metabolic syndrome at one and three years after a pregnancy complicated by preeclampsia. J Obstet Gynaecol Can 34: 836-841, 2012.

28. Spaan JJ, Sep SJ, van Balen VL, Spaanderman ME and Peeters LL: Metabolic syndrome as a risk factor for hypertension after preeclampsia. Obstet Gynecol 120: 311-317, 2012. 